\title{
A New Approach to Hyers-Ulam Stability of $r$-Variable Quadratic Functional Equations
}

\author{
Vediyappan Govindan $\mathbb{D},{ }^{1}$ Porpattama Hammachukiattikul $\mathbb{D}^{2},{ }^{2}$ Grienggrai Rajchakit $\mathbb{D}{ }^{3}$ \\ Nallappan Gunasekaran $\left(1,{ }^{4}\right.$ and R. Vadivel $\oplus^{2}$ \\ ${ }^{1}$ Department of Mathematics, DMI-St. John the Baptist University, P.O. Box 406, Mangochi, Central Africa, Malawi \\ ${ }^{2}$ Department of Mathematics, Phuket Rajabhat University, 83000 Phuket, Thailand \\ ${ }^{3}$ Department of Mathematics, Faculty of Science, Maejo University, Sansai, 50290 Chiang Mai, Thailand \\ ${ }^{4}$ Department of Mathematical Sciences, Shibaura Institute of Technology, Saitama 337-8570, Japan
}

Correspondence should be addressed to Porpattama Hammachukiattikul; porpattama@pkru.ac.th

Received 27 October 2020; Revised 22 December 2020; Accepted 9 January 2021; Published 18 January 2021

Academic Editor: Kottakkaran Sooppy Nisar

Copyright (C) 2021 Vediyappan Govindan et al. This is an open access article distributed under the Creative Commons Attribution License, which permits unrestricted use, distribution, and reproduction in any medium, provided the original work is properly cited.

In this paper, we investigate the general solution of a new quadratic functional equation of the form $\sum_{1 \leq i<j<k \leq r} \phi\left(l_{i}+l_{j}+l_{k}\right)=$ $(r-2) \sum_{i=1, i \neq j}^{r} \phi\left(l_{i}+l_{j}\right)+\left(\left(-r^{2}+3 r-2\right) / 2\right) \sum_{i=1}^{r} \phi\left(l_{i}\right)$. We prove that a function admits, in appropriate conditions, a unique quadratic mapping satisfying the corresponding functional equation. Finally, we discuss the Ulam stability of that functional equation by using the directed method and fixed-point method, respectively.

\section{Introduction}

The stability problem of functional equations originated from a question of Ulam [1] concerning about the stability. Hyers [2] gave a first affirmative answer to the question of Ulam for Banach spaces. In addition, various generalizations of Ulam's problem and Hyer's theorem have been extensively studied and many elegant results have been obtained [3-9]. The theory of nonlinear analysis has become a fastdeveloping field during the past decades. Functional equations have substantially grown to become an important branch of this field. In [10], the authors deal with a comprehensive illustration of the stability of functional equations, and in [11], the authors studied functional equations and inequalities in several variables. Very recently, most classical results on the Hyers-Ulam-Rassias stability have been offered in an integrated and self-contained version in [12]. It is worth noting that among the stability problem of functional equations, the study of the Ulam stability of different types of quadratic functional equations is an important and interesting topic, and it has attracted many scholars [13-18]. In addition, very recently, authors studied various types of stability results and have been discussed with differential equation [19-29]. To the best of the author's knowledge, a new approach to Hyers-Ulam stability of $r$-variable quadratic functional equations has not been studied so far, which motivates the present study.

Consider the functional equation as follows:

$$
\phi(l+m)+\phi(l-m)=2 \phi(l)+\phi(m),
$$

is called a quadratic functional equation. Every solution of the quadratic functional equation is a quadratic mapping. In this paper, we investigate the general solution of a new quadratic functional equation of the form

$$
\begin{aligned}
\sum_{1 \leq i<j<k \leq r} \phi\left(l_{i}+l_{j}+l_{k}\right)= & (r-2) \sum_{i=1, i \neq j}^{r} \phi\left(l_{i}+l_{j}\right) \\
& +\left(\frac{-r^{2}+3 r-2}{2}\right) \sum_{i=1}^{r} \phi\left(l_{i}\right) .
\end{aligned}
$$


Motivated by the above discussion, we prove that a function admits in appropriate conditions and a unique quadratic mapping satisfying the corresponding functional equation. Finally, we discuss the Ulam stability of that functional equation by using the directed method and fixed-point method, respectively.

\section{Preliminaries}

Definition 1. Let $l$ be a real linear space. A function $\Phi: L \times I$ $\longrightarrow[0,1]$ is said to be a fuzzy norm on $L$ if for all $l, m \in L$ and all $u, v \in I$

(N1) $\Phi(l, d)=0$ for $d \leq 0$

(N2) $l=0$ if and only if $\Phi(l, d)=1$ for all $d>0$

(N3) $\Phi(d l, v)=\Phi(l, v /|d|)$ if $d \neq 0$

(N4) $\Phi(l+m, u+v) \geq \min \{\Phi(l, u), \Phi(v, m)\}$

(N5) $\Phi(l,$.$) is a nondecreasing function on I$ and $\lim _{\nu \rightarrow \infty} \Phi$ $(l, u)=1$

(N6) for $l \neq 0, \Phi(l,$.$) is continuous on I$

The pair $(L, \Phi)$ is called fuzzy normed linear space one may regard $\Phi(l, u)$ as the truth value of the statement; the norm of $l$ is less than or equal to the real number $u$.

Definition 2. Let $(L, \Phi)$ be a fuzzy normed linear space. Let $\left\{l_{r}\right\}$ be a sequence in $L$. Then, $l_{r}$ is said to be convergent if there exists $l \in L$ such that $\lim _{r \rightarrow \infty}\left(l_{r}-l, u\right)=1$ for all $v>0$. In that case, $l$ is called the limit of the sequence $l_{r}$ and we denote it by $\Phi-\lim _{r \rightarrow \infty} l_{r}=l$.

Definition 3. A sequence $\left\{l_{r}\right\}$ be in $l$ is called Cauchy iffor each $\varepsilon>0$ and each $v>0$, there exists $r_{0}$ such that for all $r \geq r_{0}$ and all $n>0$, we have $\Phi\left(l_{r+n}-l_{r}, v\right)>1-\varepsilon$.

Definition 4. Every convergent sequence in fuzzy normed space is Cauchy. If each Cauchy sequence is convergent, then the fuzzy norm is said to be complete and the fuzzy normed space is called a fuzzy Banach space.

Theorem 5. (Banach's contraction principle). Let $(L, c)$ be a complete metric space and consider a mapping $W: L \longrightarrow L$ which is strictly contractive mapping, that is,

(A1) $c(W l, W m) \leq \mathbb{L} c(l, m)$ for some (Lipchitz constant) $\mathbb{L}<1$, then

(i) The mapping $T$ has one and only fixed point $l^{*}=W$ $\left(l^{*}\right)$

(ii) The fixed point for each given element $l^{*}$ is globally attractive that is

(A2) $\lim _{r \rightarrow \infty} W^{r} l=l^{*}$, for any starting point $l \in \mathbb{L}$

(iii) One has the following estimation inequalities: $\in \mathbb{Z}$

(A3) $c\left(W^{r} l, l^{*}\right) \leq(1 /(1-L)) c\left(W^{r} l, W^{r+1} l\right)$, for all $r \geq 0, l$

(A4) $c\left(l, l^{*}\right) \leq(1 /(1-\mathbb{L})) c\left(l, l^{*}\right)$, with respect to $l \in \mathbb{L}$
Theorem 6. (the alternative of fixed point). Suppose that for a complete generalized metric space $(L, c)$ and a strictly contractive mapping $W: L \longrightarrow M$ with Lipschitz constant $\mathbb{L}$. Then, for each given element $l \in L$, either

(B1) $c\left(W^{r} l, W^{r+1} l\right)=\infty, \forall r \geq 0$ or

(B2) there exists natural number $r_{0}$ such that:

(i) $c\left(W^{r} l, W^{l+1} r\right)<\infty$, for all $r \geq r_{0}$

(ii) The sequence $\left(W^{r} l\right)$ is convergent to a fixed point $m^{*}$ of $W$

(iii) $m^{*}$ is the unique fixed point of $W$ in the set $M=$ $\left\{m \in \mathbb{L}: c\left(W^{r} l, m\right) \infty\right\}$

(iv) $c\left(m, m^{*}\right) \leq(1 /(1-\mathbb{L})) c(v, W m)$ for all $m \in \mathbb{L}$

\section{General Solution of the Functional Equation (2)}

In this sector, the authors obtain the general solution of the functional equation (2). All over this sector, let $L$ and $M$ be real vector space.

Theorem 7. Let $L$ and $M$ be a real vector spaces. The mapping $\phi: L \longrightarrow M$ satisfies the functional equation ((2)) for all $l_{1}$, $l_{2}, l_{3}, \cdots, l_{n} \in L$, then $\phi: L \longrightarrow M$ satisfies the functional equation ((1)) for all $l, m \in L$.

Proof. We first assume that the mapping $\phi: L \longrightarrow M$ satisfies (1). Setting $l=m=0$ in (1), we get $\phi(0)=0$. Replacing $l=0$, $m=l$ in (1), then

$$
\phi(-l)=\phi(l)
$$

for all $l \in L$. Therefore, $\phi$ is even. If we choose $l=l, m=l$, and $l=2 l, m=l$ in (1), we get

$$
\phi(2 l)=4 \phi(l), \phi(3 l)=9 \phi(l)
$$

for all $l \in L$. In general for any positive integer $r$ such that

$$
\phi(r l)=r^{2} \phi(l)
$$

for all $l \in L$. Conversely, replacing $l_{1}, \cdots, l_{r}$ by $(0,0, \cdots, 0)$

in (2), we get

$$
\underbrace{(0,0, \cdot 0)}_{3 \text {-times }}
$$

$$
\phi(0)=3(r-2) \phi(0)+3\left(\frac{-r^{2}+3 r-2}{2}\right) \phi(0) .
$$

Replacing $l_{1}, \cdots, l_{r}$ by $(0,0, \cdots, 0)$ in $(2)$, we have

$$
\underbrace{}_{4-\text { times }}
$$

$$
\phi(0)=6(r-2) \phi(0)+4\left(\frac{-r^{2}+3 r-2}{2}\right) \phi(0) .
$$


Setting $l_{1}=\cdots=l_{r}$ by $(0,0, \cdots, 0)$ in $(2)$, we get

$$
\phi(0)=10(r-2) \phi(0)+5\left(\frac{-r^{2}+3 r-2}{2}\right) \phi(0) .
$$

Adding (6), (7), and (8) up to $r$-times attack, we get

$$
\begin{aligned}
\phi(0)= & \left((r-2)(3+3(r-3))+\frac{r^{2}-7 r+12}{2}\right) \phi(0) \\
& +r\left(\frac{-r^{2}+3 r-2}{2}\right) \phi(0) .
\end{aligned}
$$

It follows from (9), and using evenness of $\phi$, we get

$$
\phi(0)=0
$$

Replacing $l_{1}=\cdots=l_{r}$ by $(l,-l, 0,0, \cdots, 0)$ in (2), we obtain

$$
\begin{aligned}
\phi(0)= & (r-2)(\phi(0)+\phi(l)+\phi(-l)) \\
& +\left(\frac{-r^{2}+3 r-2}{2}\right)(\phi(l)+\phi(-l)),
\end{aligned}
$$

for all $l \in L$. Switching $l_{1}=\cdots=l_{r}$ by $(l,-l, 0,0, \cdots, 0)$ in

(2), we get

$$
\underbrace{}_{2 \text {-times }}
$$

$$
\begin{aligned}
2 \phi(0)+\phi(l)+\phi(-l)= & (r-2)(\phi(0)+2 \phi(l)+2 \phi(-l)) \\
& +\left(\frac{-r^{2}+3 r-2}{2}\right)(\phi(l)+\phi(-l)),
\end{aligned}
$$

for all $l \in L$. Setting $l_{1}=\cdots=l_{r}$ by $(l,-l, 0,0, \cdots, 0)$ in (2)

that

$$
\underbrace{}_{3-\text { times }}
$$

$$
\begin{aligned}
3 \phi(0)+3 \phi(l)+3 \phi(-l)= & (r-2)(\phi(0)+3 \phi(l)+3 \phi(-l)) \\
& +\left(\frac{-r^{2}+3 r-2}{2}\right)(\phi(l)+\phi(-l)),
\end{aligned}
$$

for all $l \in L$. Adding (11), (12), and (13), we obtain

$$
\begin{aligned}
\phi\left(l_{1}+l_{2}+l_{3}\right)= & \phi\left(l_{1}\right)+\phi\left(l_{2}\right)+\phi\left(l_{3}\right)\left((r-2)+\left(\frac{r^{2}-7 r-12}{2}\right) \phi(l)\right) \\
& +\left((r-2)+\left(\frac{r^{2}-7 r-12}{2}\right) \phi(-l)\right) \\
= & (r-2)(r-2) \phi(l)+(r-2)(r-2) \phi(-l) \\
& +\left(\frac{-r^{2}+3 r-2}{2}\right)(\phi(l)+\phi(-l)),
\end{aligned}
$$

for all $l \in L$. It follows from (14); it reduces that

$$
\phi(-l)=\phi(l)
$$

for all $l \in L$. Replacing $l_{1}=\cdots=l_{r}$ by $(l,-l, 0,0, \cdots, 0)$ in

(2), we get

$$
\phi(2 l)=(r-2)(\phi(2 l)+2 \phi(l))+\left(\frac{-r^{2}+3 r-2}{2}\right)(2 \phi(l)),
$$

for all $l \in L$. Substituting $l_{1}=\cdots=l_{r}$ by $(l, l, 0,0, \cdots, 0)$ in

(2), we arrive

$$
\underbrace{}_{\text {2-times }}
$$

$$
2 \phi(2 l)+2 \phi(l)=(r-2)(\phi(2 l)+4 \phi(l))+\left(\frac{-r^{2}+3 r-2}{2}\right)(2 \phi(l))
$$

for all $l \in L$. Replacing $l_{1}=\cdots=l_{r}$ by $(l, l, 0,0, \cdots, 0)$ in

(2), we get

$$
\underbrace{0}_{\text {3-times }}
$$

$3 \phi(2 l)+6 \phi(l)=(r-2)(\phi(2 l)+6 \phi(l))+\left(\frac{-r^{2}+3 r-2}{2}\right)(2 \phi(l))$,

for all $l \in L$. Adding (16), (17), and (18) using evenness of $\phi$, then we get

$$
\begin{aligned}
(r-2) & \phi(2 l)+\left(2(r-3)+\left(r^{2}-7 r+12\right) \phi(l)\right) \\
& =(r-2)(\phi(2 l)+(2 r-4) \phi(l))+\left(\frac{-r^{2}+3 r-2}{2}\right)(2 \phi(l)),
\end{aligned}
$$

for all $l \in L$. It follows from (19); we get

$$
\phi(2 l)=4 \phi(l)
$$

for all $l \in L$. In general for any positive integer $r$, then can be written as

$$
\phi(r l)=r^{2} \phi(l)
$$

for all $l \in L$. Replacing $l_{1}=\cdots=l_{r}$ by $(l, m, 0,0, \cdots, 0)$, we

arrive 


$$
\begin{aligned}
(r-2) \phi( & +m)+\left(\left((r-3)+\left(\frac{r^{2}-7 r+12}{2}\right)\right) \phi(l)\right. \\
& \left.+\left((r-3)+\left(\frac{r^{2}-7 r+12}{2}\right)\right) \phi(m)\right) \\
& \cdot(r-2)(\phi(l+m)+(r-2) \phi(l)+(r-2) \phi(m)) \\
& +\left(\frac{-r^{2}+3 r-2}{2}\right)(2 \phi(l)+\phi(m)),
\end{aligned}
$$

for all $l, m \in L$. Setting $l_{1}=\cdots=l_{r}$ by $(l,-l, m, 0,0, \cdots, 0)$

$$
\underbrace{0}_{(r-3) \text {-times }}
$$$$
\phi(m)=(r-2)(\phi(l+m)+\phi(-l+m))
$$$$
+\left(\frac{-r^{2}+3 r-2}{2}\right)(\phi(l)+\phi(-l)+\phi(m)) \text {, }
$$

for all $l, m \in L$. Replacing $l_{1}=\cdots=l_{r}$ by $(l,-l, m$,

$0,0, \cdots, 0)$ in $(2)$, we get

$$
\begin{aligned}
& \underbrace{\underbrace{2}}_{(r-3) \text {-times }} \\
& \phi(m)+\phi(l+m)+\phi(l+m) \\
& =(l-2)(\phi(l+m)+\phi(-l+m)+2 \phi(l)+2 \phi(m)) \\
& +\left(\frac{-r^{2}+3 r-2}{2}\right)(\phi(l)+\phi(-l)+\phi(m)) \text {, }
\end{aligned}
$$

for all $l, m \in L$. Substituting $l_{1}=\cdots=l_{r}$ by $(l,-l, m$,

$0,0, \cdots, 0)$ in (2) that

$\underbrace{}_{(r-3) \text {-times }}$

$$
\begin{aligned}
& 2 \phi(m)+2 \phi(l+m)+2 \phi(-l+m)+2 \phi(l) \\
& =(r-2)(\phi(l+m)+\phi(-l+m)+4 \phi(l)+2 \phi(m)) \\
& \quad+\left(\frac{-r^{2}+3 r-2}{2}\right)(\phi(l)+\phi(-l)+\phi(m)),
\end{aligned}
$$

for all $l, m \in L$. Adding (23), (24), and (25) and using evenness of $\phi$, we get

$$
\begin{aligned}
(1 & \left.+\left(\frac{r^{2}-7 r+12}{2}\right)\right) \phi(m)+\left(r^{2}-7 r+12\right) \phi(l)+2 \phi(l+m) \\
& +(r-2) \phi(-l+m)+(r-2) \phi(l)=(r-2)(\phi(l+m)+\phi(-l+m)) \\
& +(r-2)(2 r-6) \phi(l)+(r-2)(r-3) \phi(m) \\
& +\left(\frac{-r^{2}+3 r-2}{2}\right)(\phi(l)+\phi(-l)+\phi(m)),
\end{aligned}
$$

for all $l, m \in L$. Replace $m$ by $-m$ in (26), we get

$$
\begin{aligned}
(1 & \left.+\left(\frac{r^{2}-7 r+12}{2}\right)\right) \phi(-m)+\left(r^{2}-7 r+12\right) \phi(l)+2 \phi(l-m) \\
& +(r-2) \phi(-l-m)+(r-2) \phi(l)=(r-2)(\phi(l-m)+\phi(-l-m)) \\
& +(r-2)(2 r-6) \phi(l)+(r-2)(r-3) \phi(-m) \\
& +\left(\frac{-l^{2}+3 l-2}{2}\right)(\phi(l)+\phi(-l)+\phi(-m)),
\end{aligned}
$$

for all $l, m \in L$. Adding (26) and (27) and using evenness of $\phi$, then

$$
\phi(l+m)+\phi(l-m)=2 \phi(l)+2 \phi(m)
$$

for all $l, m \in L$. So the mapping $\phi: L \longrightarrow M$ is quadratic.

In Sections 4 and 5, using $L$ be a normed space and $M$ be a Banach space. For notational handiness, we define a function $D \phi: L \longrightarrow M$ by

$$
\begin{aligned}
D \phi\left(l_{1}, l_{2}, \cdots l_{r}\right)= & \sum_{1 \leq i<j<k \leq r} \phi\left(l_{i}+l_{j}+l_{k}\right)-(r-2) \sum_{i=1, i \neq j}^{r} \phi\left(l_{i}+l_{j}\right) \\
& +\left(\frac{-r^{2}+3 r-2}{2}\right) \sum_{i=1}^{r} \phi\left(l_{i}\right),
\end{aligned}
$$

for all $l_{1}, l_{2}, \cdots l_{r} \in L$.

\section{Stability of the Functional Equation (2): Direct Method}

In this section, we establish the stability of (2) in a fuzzy Banach space using a direct method.

Theorem 8. Let $\beta \in\{-1,1\}$. Let $\chi: L^{r} \longrightarrow N$ be a mapping with

$$
0<\left(\frac{c}{3^{2}}\right)<1 \Phi^{\prime}\left(\chi\left(3^{\beta \kappa} l_{1}, 3^{\beta \kappa} l_{2}, \cdots, 3^{\beta \kappa} l_{r}\right), n\right) \geq \Phi^{\prime}\left(c^{\beta} \chi(l, l, \cdots, l), n\right),
$$

for all $l \in L$ and all $n>0, c>0$ and

$$
\lim _{\kappa \rightarrow} \Phi^{\prime}\left(\chi\left(3^{\beta \kappa} l_{1}, 3^{\beta \kappa} l_{2}, \cdots, 3^{\beta \kappa} l_{r}\right), 3^{\beta \kappa} n\right)=1,
$$

for all $l_{1}, l_{2}, \cdots, l_{r} \in L$ and all $n>0$. Suppose that a function $D \phi: L \longrightarrow M$ satisfies the inequality

$$
\Phi\left(D \phi\left(l_{1}, l_{2}, \cdots, l_{r}\right), n\right) \geq \Phi^{\prime}\left(\chi\left(l_{1}, l_{2}, \cdots, l_{r}\right), n\right),
$$

for all $n>0$ and $l_{1}, l_{2}, \cdots, l_{r} \in L$. Then, the limit

$$
Q(l)=\Phi-\lim _{\kappa \rightarrow \infty} \frac{\phi\left(3^{\beta \kappa} l\right)}{3^{\beta \kappa}}
$$


exists for all $l \in L$ and the mapping $Q: L \longrightarrow M$ is a unique quadratic mapping such that

$\phi(\phi(l)-Q(l), n) \geq \Phi^{\prime}\left(\chi(l, l, \cdots, l), \frac{3\left(r^{3}-3 r^{2}+2 r\right)}{2} n\left|3^{2}-c\right|\right)$,

for all $l \in L$ and for all $n>0$.

Proof. First, assume that $\beta=1$. Replacing $\left(l_{1}, l_{2}, \cdots, l_{r}\right)$ by $(l, l, \cdots, l)$, in $(32)$, we have

$\Phi\left(\left(\frac{\phi\left(r^{3}-3 r^{2}+2 r\right)}{6} \phi(3 l)-\frac{3\left(r^{3}-3 r^{2}+2 r\right)}{2} \phi(l)\right), n\right) \geq \Phi^{\prime}(\chi(l, l, \cdots, l), n)$,

for all $l \in L$ and for all $n>0$. Replacing $l$ by $3^{\kappa} l$ in (35), we obtain

$\Phi\left(\frac{\phi\left(3^{\kappa+1} l\right)}{3^{2}}-\phi\left(3^{\kappa} l\right), \frac{2 n}{3\left(r^{3}-3 r^{2}+2 r\right)}\right) \geq \Phi^{\prime}\left(\chi\left(3^{\kappa} l, 3^{\kappa} l, \cdots, 3^{\kappa} l\right), n\right)$,

for all $l \in L$ and for all $n>0$. Using (30) and (N3) in (36), we have

$$
\Phi\left(\frac{\phi\left(3^{\kappa+1} l\right)}{3^{2}}-\phi\left(3^{\kappa} l\right), \frac{2 n}{3\left(r^{3}-3 r^{2}+2 r\right)}\right) \geq \Phi^{\prime}\left(\chi\left(3^{\kappa} l, 3^{\kappa} l, \cdots, 3^{\kappa} l\right), \frac{n}{c^{\kappa}}\right),
$$

for all $l \in L$ and for all $n>0$; it is easy to verify from (37) that

$\Phi\left(\frac{\phi\left(3^{\kappa+1} l\right)}{3^{2(\kappa+1)}}-\frac{\phi\left(3^{\kappa} l\right)}{3^{2 \kappa}}, \frac{2 n}{3\left(r^{3}-3 r^{2}+2 r\right) 3^{2 l}}\right) \geq \Phi^{\prime}\left(\chi\left(3^{\kappa} l, 3^{\kappa} l, \cdots, 3^{\kappa} l\right), \frac{n}{c^{\kappa}}\right)$,

holds for all $l \in L$ and for all $n>0$. Replacing $n$ by $c^{\kappa} n$ in (38), we get

$\Phi\left(\frac{\phi\left(3^{\kappa+1} l\right)}{3^{2(\kappa+1)}}-\frac{\phi\left(3^{\kappa} l\right)}{3^{2 \kappa}}, \frac{2 v^{\kappa} n}{3\left(r^{3}-3 r^{2}+2 r\right) 3^{2 \kappa}}\right) \geq \Phi^{\prime}\left(\chi\left(3^{\kappa} l, 3^{\kappa} l, \cdots, 3^{\kappa} l\right), n\right)$,

for all $l \in L$ and for all $n>0$; it is easy to see that

$$
\frac{\phi\left(3^{\kappa+1} l\right)}{3^{2(\kappa+1)}}-\phi(l)=\sum_{i=0}^{\kappa-1}\left[\frac{\phi\left(3^{i+1} l\right)}{3^{2(i+1)}}-\frac{\left(\Phi 3^{i} x\right)}{3^{2 i}}\right],
$$

for all $l \in L$. From equations (39) and (40), we get

$$
\begin{gathered}
\Phi\left(\frac{\phi\left(3^{\kappa} l\right)}{3^{2 \kappa}}-\phi(l), \sum_{i=0}^{\kappa-1} \frac{2 c^{i} n}{3\left(r^{3}-3 r^{2}+2 r\right) 3^{2 i}}\right) \\
\geq \min \bigcup_{i=1}^{\kappa-1}\left\{\frac{\phi\left(3^{i+1} l\right)}{3^{2(i+1)}}-\frac{\phi\left(3^{i} l\right)}{3^{2 i}}, \frac{2 c^{i} n}{3\left(r^{3}-3 r^{2}+2 r\right) 3^{2 i}}\right\} \\
\geq \min \bigcup_{i=1}^{\kappa-1} \Phi^{\prime}(\chi(l, l, \cdots, l), n) \geq \Phi^{\prime}(\chi(l, l, \cdots, l), n),
\end{gathered}
$$

for all $l \in L$ and for all $n>0$. Replacing $l$ by $3^{m} l$ in (41) and using (30) and (N3), we obtain

$$
\begin{aligned}
& \Phi\left(\frac{\phi\left(3^{\kappa+m} l\right)}{3^{2(\kappa+m)}}-\frac{\phi\left(3^{m} l\right)}{3^{2 m}}, \sum_{i=0}^{m+\kappa-1} \frac{2 c^{i} n}{3\left(r^{3}-3 r^{2}+2 r\right) 3^{2 i}}\right) \\
& \geq \Phi^{\prime}\left(\chi(l, l, \cdots, l), \frac{n}{c^{m}}\right),
\end{aligned}
$$

for all $l \in L$ and for all $n>0$. And all $m, \kappa \geq 0$. Replacing $n$ by $c^{m} n$ in (42), we get

$$
\begin{aligned}
& \Phi\left(\frac{\phi\left(3^{\kappa+m} l\right)}{3^{2(\kappa+m)}}-\frac{\phi\left(3^{m} l\right)}{3^{2 m}}, \sum_{i=m}^{m+\kappa-1} \frac{2 c^{i} n}{3\left(r^{3}-3 r^{2}+2 r\right) 3^{2 i}}\right) \\
& \geq \Phi^{\prime}(\chi(l, l, \cdots, l), n),
\end{aligned}
$$

for all $l \in L$ and for all $n>0$. And all $m, \kappa \geq 0$. Using (N3) in (42), we have

$$
\begin{aligned}
& \Phi\left(\frac{\phi\left(3^{\kappa+m} l\right)}{3^{2(\kappa+m)}}-\frac{\phi\left(3^{m} l\right)}{3^{2 m}}, n\right) \\
& \quad \geq \Phi^{\prime}\left(\chi(l, l, \cdots, l), \frac{n}{\sum_{i=m}^{m+\kappa-1}\left(2 c^{i} / 3\left(r^{3}-3 r^{2}+2 r\right) 3^{2 i}\right)}\right)
\end{aligned}
$$

for all $l \in L$ and for all $n>0$. And all $m, \kappa \geq 0$. Since $0<c<3^{2}$ and $\sum_{i=0}^{\kappa}\left(c / 3^{2}\right)^{i}<\infty$, the Cauchy criterion for convergence and (N5) implies that $\left\{\phi\left(3^{\kappa} l\right) / 3^{2 \kappa}\right\}$ is a Cauchy sequence in $\left(M, \Phi^{\prime}\right)$ is a fuzzy Banach space. This sequence converges to some point $Q(l) \in M$ so one can define the mapping $Q: L \longrightarrow M$ by

$$
Q(l)=\Phi-\lim _{\kappa \rightarrow \infty} \frac{\phi\left(3^{\beta \kappa} l\right)}{3^{2 \beta \kappa}},
$$

for all $l \in L$. Letting $m=0$ in (44), we receive

$$
\begin{aligned}
& \Phi\left(\frac{\phi\left(3^{\kappa} l\right)}{3^{2 \kappa}}-\phi(l), n\right) \\
& \quad \geq \Phi^{\prime}\left(\chi(l, l, \cdots, l), \frac{n}{\sum_{i=0}^{\kappa-1}\left(c^{i} n / 3\left(r^{3}-3 r^{2}+2 r\right) 3^{2 i}\right)}\right),
\end{aligned}
$$


for all $l \in L$. Letting $\kappa \longrightarrow \infty$ in (46) and using (N6), we have

$\phi(\phi(t)-Q(l), n) \geq \Phi^{\prime}\left(\chi(l, l, \cdots, l), \frac{3\left(r^{3}-3 r^{2}+2 r\right)}{2} n\left(3^{2}-c\right)\right)$,

for all $l \in L$ and for all $n>0$. To prove $Q$ satisfies (2), replacing $\left(l_{1}, l_{2}, \cdots, l_{r}\right)$ by $\left(3{ }^{\kappa} l_{1}, 3{ }^{\kappa} l_{2}, \cdots, 3^{\kappa} l_{r}\right)$ in (32), we get

$$
\begin{aligned}
& \Phi\left(\frac{1}{3^{\kappa}} D \phi\left(3^{\kappa} l_{1}, 3^{\kappa} l_{2}, \cdots, 3^{\kappa} l_{r}\right), r\right) \\
& \quad \geq \Phi^{\prime}\left(\chi\left(3^{\kappa} l_{1}, 3^{\kappa} l_{2}, \cdots, 3^{\kappa} l_{r}\right), 3^{2 \kappa} n\right),
\end{aligned}
$$

for all $n>0$ and all $l_{1}, l_{2}, \cdots, l_{r} \in L$, since $\lim _{\kappa \rightarrow \infty} \Phi^{\prime}\left(\chi\left(3^{\beta \kappa} l_{1}\right.\right.$, $\left.\left.3^{\beta \kappa} l_{2}, \cdots, 3^{\beta \kappa} l_{r}\right), 3^{2 \beta \kappa} n\right)=1$.

Hence, $Q$ satisfies the quadratic functional equation (2), in order to prove $Q(l)$ is unique.

We let $R(l)$ be another quadratic functional equation satisfying (2) and (34). Hence,

$$
\begin{aligned}
\phi(Q(l)-R(l), n) & =\Phi\left(\frac{Q\left(3^{\kappa} l\right)}{3^{2 \kappa}}-\frac{R\left(3^{\kappa} l\right)}{3^{2 \kappa}}\right) \\
& \geq \min \left\{\Phi\left(\frac{Q\left(3^{\kappa} l\right)}{3^{2 \kappa}}-\frac{\phi\left(3^{\kappa} l\right)}{3^{2 \kappa}}, \frac{n}{2}\right), \Phi\left(\frac{\phi\left(3^{\kappa} l\right)}{3^{2 \kappa}}-\frac{R\left(3^{\kappa} l\right)}{3^{2 \kappa}}, \frac{n}{2}\right)\right\} \\
& \geq \Phi^{\prime}\left(\chi\left(3^{\kappa} l, 3^{\kappa} l, \cdots, 3^{\kappa} l\right) \frac{\left(r^{3}-3 r^{2}+2 r\right) 3^{2 \kappa} n\left(3^{2}-c\right)}{4}\right) \\
& \geq \Phi\left(\chi(l, l, \cdots, l), \frac{3\left(r^{3}-3 r^{2}+2 r\right) 3^{2 \kappa} n\left(3^{2}-c\right)}{4 c^{\kappa}}\right),
\end{aligned}
$$

for all $l \in L$ and for $n>0$. Since

$$
\lim _{\kappa \rightarrow \infty} \frac{3\left(r^{3}-3 r^{2}+2 r\right) 3^{2 \kappa} n\left(3^{2}-c\right)}{4 c^{\kappa}}=\infty
$$

we obtain

$$
\Phi^{\prime}\left(\chi\left(3^{\kappa} l, 3^{\kappa} l, \cdots, 3^{\kappa} l\right), \frac{3\left(r^{3}-3 r^{2}+2 r\right) 3^{2 \kappa} n\left(3^{2}-c\right)}{4 c^{\kappa}}\right)=1 .
$$

Thus, $\Phi(Q(l)-R(l), n)=1$ for all $l \in L$ and for $n>0$. Hence, $Q(l)=R(l)$. Therefore, $Q(l)$ is unique. For $\beta=-1$, we can prove the result by a similar method. This completes the proof of the theorem.

The following Corollary 9 is an immediate consequence of Theorem 8 concerning the stability of (2).
Corollary 9. Suppose that the function $D \phi: L \longrightarrow M$ satisfies the inequality

$$
\phi\left(D \phi\left(l_{1}, l_{2}, \cdots, l_{r}\right), n\right) \geq\left\{\begin{array}{l}
\Phi^{\prime}(\varepsilon, n), \\
\Phi^{\prime}\left(\varepsilon \sum_{i=1}^{r}\left\|l_{i}\right\|^{s}, n\right), \\
\Phi^{\prime}\left(\varepsilon \prod_{i=1}^{n}\left\|l_{i}\right\|^{s}, r\right), \\
\Phi^{\prime}\left(\varepsilon\left(\sum_{i=1}^{r}\left\|l_{i}\right\|^{r s}+\prod_{i=1}^{r}\left\|l_{i}\right\|^{s}\right), n\right),
\end{array}\right.
$$

for all $l_{1}, l_{2}, \cdots, l_{r} \in L$ and all $n>0$, where $\varepsilon, s$ are constants. Then, there exists a unique quadratic mapping $Q: L \longrightarrow M$ such that

$$
\phi(\phi(t)-Q(l), n) \geq\left\{\begin{array}{l}
\Phi^{\prime}\left(\varepsilon, \frac{3\left(r^{3}-3 r^{2}+2 r\right)}{2} n|8|\right), \\
\Phi^{\prime}\left(r \varepsilon\|l\|^{s}, \frac{3\left(r^{3}-3 r^{2}+2 r\right)}{2} m\left|3^{2}-3^{s}\right|\right) ; s \neq 2, \\
\Phi^{\prime}\left(\varepsilon\|l\|^{r s}, \frac{3\left(r^{3}-3 r^{2}+2 r\right)}{2} n\left|3^{2}-3^{r s}\right|\right) ; s \neq \frac{2}{r}, \\
\Phi^{\prime}\left(\varepsilon(r+1)\|l\|^{r s}, \frac{3\left(r^{3}-3 r^{2}+2 r\right)}{2} n\left|3^{2}-3^{r s}\right|\right) ; s \neq \frac{2}{r},
\end{array}\right.
$$

for all $l \in L$ and for $n>0$.

\section{Stability of the Functional Equation (2): Fixed-Point Method}

In this section, the authors investigate the generalized UlamHyers stability of the functional equation (2) in fuzzy normed space using the fixed-point method.

To prove the stability result, we define the following $\psi_{i}$ is a constant such that

$$
\psi_{i}= \begin{cases}3 & \text { if } i=0 \\ \frac{1}{3} & \text { if } i=1\end{cases}
$$

and $\Omega$ is the set such that $\omega=\{u \backslash u: L \longrightarrow M, u(0)=0\}$.

Theorem 10. Let $D \phi: L \longrightarrow M$ be a mapping for which there exists a function $\chi: L^{r} \longrightarrow N$ with condition

$$
\lim _{\kappa \rightarrow \infty} \Phi^{\prime}\left(\chi\left(\psi_{i}^{\kappa} l_{1}, \psi_{i}^{\kappa} l_{2}, \cdots, \psi_{i}^{\kappa} l_{r}\right), \psi_{i}^{\kappa} n\right)=1
$$

for all $l_{1}, l_{2}, \cdots, l_{r} \in L, n>0$ and satisfying the inequality

$$
\phi\left(D \phi\left(l_{1}, l_{2}, \cdots, l_{r}\right), n\right) \geq \Phi^{\prime}\left(D \phi\left(l_{1}, l_{2}, \cdots, l_{r}\right), n\right),
$$

for all $l_{1}, l_{2}, \cdots, l_{r} \in L$ and $n>0$. 
If there exists $\mathbb{L}=\mathbb{L}[i]$ such that the function

$$
l \longrightarrow \rho(l)=\frac{2}{\left(r^{3}-3 r^{2}+2 r\right)} \chi\left(\frac{l}{3}, \frac{l}{3}, \cdots, \frac{l}{3}\right)
$$

has the property

$$
\Phi^{\prime}\left(\mathbb{L} \frac{i}{\psi_{i}^{2}} \rho\left(\psi_{i} l\right), n\right)=\Phi^{\prime}(\rho(l), n)
$$

for all $l \in L$ and $n>0$. Then, there exists a unique quadratic function $Q: L \longrightarrow M$ satisfying the functional equation (2) and

$$
\phi(\phi(t)-Q(l), n) \geq \Phi^{\prime}\left(\frac{\mathbb{L}^{1-i}}{1-\mathbb{L}} \rho(l), n\right)
$$

for all $l \in L$ and $n>0$.

Proof. Let $c$ be a general metric on $\Omega$, such that

$c(u, v)=\inf \left\{\kappa \in \frac{0, \infty}{\phi(u(l)-v(l), n)} \geq \Phi^{\prime}(\rho(l), \kappa n), l \in L, l>0\right\}$.

It is easy to see that $\Omega, c$ is complete.

Define $W: \Omega \longrightarrow \Omega$ by $W(l)=\left(1 / \psi_{i}^{2}\right) u\left(\psi_{i} l\right), \forall l \in L$.

For $u, v \in \Omega$, we get

$$
\begin{aligned}
c(u, v) \leq \kappa & \Longrightarrow \phi(u(l)-v(l), n) \geq \Phi^{\prime}(\rho(l), \kappa n) \\
& \Longrightarrow \Phi\left(\frac{u\left(\psi_{i} l\right)}{\psi_{i}^{2}}-\frac{v\left(\psi_{i} l\right)}{\psi_{i}^{2}}, n\right) \geq \Phi^{\prime}\left(\rho\left(\psi_{i} l\right), \kappa \psi_{i}^{2} n\right) \\
& \Longrightarrow \phi(W u(l)-W v(l), n) \geq \Phi^{\prime}\left(\rho\left(\psi_{i} l\right), \kappa \psi_{i}^{2} n\right) \\
& \Longrightarrow \phi(W u(l)-W v(l), n) \geq \Phi^{\prime}(\rho(l), \kappa \llbracket n) \\
& \Longrightarrow c(W u(l), W v(l), n) \leq \kappa \llbracket n \\
& \Longrightarrow c(W u, W v, n) \leq \kappa c(u, v), \quad \forall u, v \in \Omega .
\end{aligned}
$$

Therefore, $W$ is strictly contractive mapping on $\Omega$ with Lipschitz constant $\mathbb{L}$, replacing $\left(l_{1}, l_{2}, \cdots, l_{r}\right)$ by $(l, l, \cdots, l)$ in (56), we get

$$
\begin{aligned}
& \Phi\left(\frac{3\left(r^{3}-3 r^{2}+2 r\right)}{6} \phi(3 l)-\frac{3\left(r^{3}-3 r^{2}+2 r\right)}{2} \phi(l), n\right) \\
& \quad \geq \Phi^{\prime}(\chi(l, l, \cdots, l), n),
\end{aligned}
$$

for all $l \in L$ and $n>0$. Using (N3) in (62), we have

$\Phi\left(\frac{\phi(3 l)}{3^{2}}-\phi(t), n\right) \geq \Phi^{\prime}\left(\frac{2}{3\left(r^{3}-3 r^{2}+2 r\right)} \chi(l, l, \cdots, l), n\right)$, for all $l \in L$ and $n>0$ with the help of (58), when $i=0$. It follows from (63) that

$$
\Longrightarrow \Phi\left(\frac{\phi(3 l)}{3^{2}}-\phi(t), n\right) \geq \Phi^{\prime}(\mathbb{L} \rho(l), n) \Longrightarrow c(W \phi, \phi) \leq \mathbb{L}_{1-i} .
$$

Replacing $l$ by $l / 3$ in (62), we get

$$
\begin{aligned}
& \Phi\left(\phi(l)-3^{2} \phi\left(\frac{l}{3}\right), n\right) \\
& \left.\quad \geq \Phi^{\prime}\left(\frac{2}{3\left(r^{3}-3 r^{2}+2 r\right)}\right) \times\left(\frac{l}{3}, \frac{l}{3}, \cdots, \frac{l}{3}\right), n\right),
\end{aligned}
$$

for all $l \in L$ and $n>0$ when $i=1$; it follows from (65); we arrive

$$
\Longrightarrow \Phi\left(\phi(l)-3^{2} \phi\left(\frac{l}{3}\right), n\right) \geq \Phi^{\prime}(\rho(l), n) \Longrightarrow c(\phi, W \phi) \leq \mathbb{L}^{1-i} .
$$

Then from (64) and (66), we get

$$
\Longrightarrow c(\phi, W \phi) \leq \mathbb{L}^{1-i} \leq \infty .
$$

Now from the fixed-point alternative in both cases, it follows that there exists a fixed point $Q$ of $W$ in $\Omega$ such that

$$
Q(x)=\Phi-\lim _{\kappa \rightarrow \infty} \frac{\phi\left(\psi^{\kappa} l\right)}{\psi^{2 \kappa}},
$$

for all $l \in L$ and $n>0$. Replacing $\left(l_{1}, l_{2}, \cdots, l_{r}\right)$ by $\left(\psi_{i}^{\kappa} l_{1}\right.$ , $\left.\psi_{i}^{\kappa} l_{2}, \cdots, \psi_{i}^{\kappa} l_{r}\right)$ in (56), we get

$$
\begin{aligned}
& \Phi\left(\frac{1}{\psi_{i}^{2 \kappa}} D \phi\left(\psi_{i}^{\kappa} l_{1}, \psi_{i}^{\kappa} l_{2}, \cdots, \psi_{i}^{\kappa} l_{r}\right), n\right) \\
& \quad \geq \Phi^{\prime}\left(\chi\left(\psi_{i}^{\kappa} l_{1}, \psi_{i}^{\kappa} l_{2}, \cdots, \psi_{i}^{\kappa} l_{r}\right), \psi_{i}^{2 \kappa} n\right),
\end{aligned}
$$

for all $n>0$ and all $l_{1}, l_{2}, \cdots, l_{r} \in L$. Utilizing the same procedure in Theorem 8 , we can prove the function $Q: L$ $\longrightarrow M$ is quadratic and it satisfies the functional equation (2) by a fixed-point alternative, since $Q$ is a unique fixed point of $W$ in the set $\Delta=\{\phi \in \Omega / c(\phi, Q)<\infty\}$. Therefore, $Q$ is a unique function such that

$$
\phi(\phi(t)-Q(l), n) \geq \Phi^{\prime}(\rho(l), \kappa n),
$$

for all $l \in L$ and $n>0$. Again using the fixed-point alternative, we get 


$$
\begin{aligned}
c(\phi, Q) & \leq \frac{1}{1-\mathbb{L}} c(\phi, W \phi) \Longrightarrow c(\phi, Q) \leq \frac{\mathbb{L}^{1-i}}{1-\mathbb{L}} \\
& \Longrightarrow \phi(\phi(t)-Q(l), n) \geq \Phi^{\prime}\left(\rho(l) \frac{\mathbb{L}^{1-i}}{1-\mathbb{L}}, n\right) .
\end{aligned}
$$

This completes the proof.

The following Corollary 11 is an immediate consequence of Theorem 10 concerning the stability of (2).

Corollary 11. Suppose that the function $D \phi: L \longrightarrow M$ satisfies the inequality

$$
\phi\left(D \phi\left(l_{1}, l_{2}, \cdots, l_{r}\right), n\right) \geq\left\{\begin{array}{l}
\Phi^{\prime}(\varepsilon, n), \\
\Phi^{\prime}\left(\varepsilon\left\{\sum_{i=1}^{r}\left\|l_{i}\right\|^{s}\right\}, n\right), \\
\Phi^{\prime}\left(\varepsilon\left\{\prod_{i=1}^{r}\left\|l_{i}\right\|^{s}\right\}, n\right), \\
\Phi^{\prime}\left(\varepsilon\left\{\sum_{i=1}^{r}\left\|l_{i}\right\|^{n s}+\prod_{i=1}^{r}\left\|l_{i}\right\|^{s}\right\}, n\right),
\end{array}\right.
$$

for all $l_{1}, l_{2}, \cdots, l_{r} \in L$ and all $n>0$, where $\varepsilon, s$ are constants. Then, there exists a unique quadratic mapping $A: L$ $\longrightarrow M$ such that

$$
\phi(\phi(l)-A(l), n) \geq\left\{\begin{array}{l}
\Phi^{\prime}\left(\varepsilon, \frac{3\left(r^{3}-3 r^{2}+2 r\right)}{2} n|8|\right), \\
\Phi^{\prime}\left(r \varepsilon\left|\left\|l_{i}\right\|^{s}, \frac{3\left(r^{3}-3 r^{2}+2 r\right)}{2} n\right| 3^{2}-3^{s} \mid\right) ; s \neq 2, \\
\Phi^{\prime}\left(\varepsilon\left\|l_{i}\right\|^{r s}, \frac{3\left(r^{3}-3 r^{2}+2 r\right)}{2} n\left|3^{2}-3^{r s}\right|\right) ; s \neq \frac{2}{r}, \\
\Phi^{\prime}\left(\varepsilon(r+1)\left\|l_{i}\right\|^{r s}, \frac{3\left(r^{3}-3 r^{2}+2 r\right)}{2} n\left|3^{2}-3^{r s}\right|\right) ; s \neq \frac{2}{r},
\end{array}\right.
$$

for all $l \in L$ and for $n>0$.

Proof. Setting

$$
\chi\left(l_{1}, l_{2}, \cdots, l_{r}\right) \leq\left\{\begin{array}{l}
\varepsilon\left\{\sum_{i=1}^{\varepsilon}\left\|l_{i}\right\|^{s}\right\}, \\
\varepsilon\left\{\prod_{i=1}^{r}\left\|l_{i}\right\|^{s}\right\}, \\
\varepsilon\left\{\sum_{i=1}^{r}\left\|l_{i}\right\|^{r s}+\prod_{i=1}^{r}\left\|l_{i}\right\|^{s}\right\},
\end{array}\right.
$$

for all $l_{1}, l_{2}, \cdots, l_{r} \in L$. Then,

$$
\begin{aligned}
& \Phi^{\prime}\left(\chi\left(\psi_{i}^{\kappa} l_{1}, \psi_{i}^{\kappa} l_{2}, \cdots, \psi_{i}^{\kappa} l_{r}\right), \psi_{i}^{2 \kappa} n\right) \\
& =\left\{\begin{array}{l}
\Phi^{\prime}\left(\varepsilon, \psi_{i}^{K} n\right), \\
\Phi^{\prime}\left(\varepsilon\left\{\sum_{i=1}^{r}\left\|l_{i}\right\|^{s}\right\}, \psi_{i}^{(2-s) K} n\right), \\
\left.\Phi^{\prime}\left\{\sum_{i=1}^{r}\left\|l_{i}\right\|^{s}\right\}, \psi_{i}^{(2-r s) K} n\right), \\
\Phi^{\prime}\left(\varepsilon\left\{\sum_{i=1}^{r}\left\|l_{i}\right\|^{r s}+\prod_{i=1}^{r}\left\|l_{i}\right\|^{s}\right\}, \psi_{i}^{(2-r s) K} n\right), \\
\longrightarrow 1 \text { as } \kappa \longrightarrow \infty, \\
\longrightarrow 1 \text { as } \kappa \longrightarrow \infty,
\end{array}\right. \\
& \quad\left\{\begin{array}{l}
\longrightarrow 1 \text { as } \kappa \longrightarrow \infty, \\
\longrightarrow \infty,
\end{array}\right.
\end{aligned}
$$

i.e., (55) holds. We have $\rho(l)=\left(2 / 3\left(r^{3}-3 r^{2}+2 r\right)\right) \chi(l / 3, l / 3$, $\cdots, l / 3)$ that has the property $\Phi^{\prime}\left(\mathbb{L}\left(1 / \psi_{i}^{2}\right) \rho\left(\psi_{i} l\right), n\right)=\Phi^{\prime}(\rho$ $(l), n)$ for all $l \in L$ and $n>0$. Hence,

$$
\begin{aligned}
\Phi^{\prime}(\rho(l), n) & =\Phi^{\prime}\left(\chi\left(\frac{l}{3}, \frac{l}{3}, \cdots, \frac{l}{3}\right), \frac{3\left(r^{3}-3 r^{2}+2 r\right)}{2} n\right) \\
& =\left\{\begin{array}{l}
\Phi^{\prime}\left(\varepsilon, \frac{3\left(r^{3}-3 r^{2}+2 r\right)}{2} n\right), \\
\Phi^{\prime}\left(r \varepsilon\|l\|^{s}, \frac{3\left(r^{3}-3 r^{2}+2 r\right)}{2} 3^{s} n\right), \\
\Phi^{\prime}\left(\varepsilon\|l\|^{r s}, \frac{3\left(r^{3}-3 r^{2}+2 r\right)}{2} 3^{r s} n\right), \\
\Phi^{\prime}\left(\varepsilon(r+1)\|l\|^{r s}, \frac{3\left(r^{3}-3 r^{2}+2 r\right)}{2} 3^{r s} n\right) .
\end{array}\right.
\end{aligned}
$$

Now,

$$
\Phi^{\prime}\left(\frac{1}{\psi_{i}^{2}} \rho\left(\psi_{i} l\right), n\right)=\left\{\begin{array}{l}
\Phi^{\prime}\left(\frac{\varepsilon}{\psi_{i}^{2}}, \frac{3\left(r^{3}-3 r^{2}+2 r\right)}{2} n\right), \\
\Phi^{\prime}\left(\frac{r \varepsilon\|l\|^{s} \psi_{i}^{s}}{\psi_{i}^{2} 3^{s}}, \frac{3\left(r^{3}-3 r^{2}+2 r\right)}{2} n\right), \\
\Phi^{\prime}\left(\frac{\varepsilon\|l\|^{r s} \psi_{i}^{r s}}{\psi_{i}^{2} 3^{r s}}, \frac{3\left(r^{3}-3 r^{2}+2 r\right)}{2} n\right), \\
\Phi^{\prime}\left(\frac{(r+1) \varepsilon\|l\|^{r s} \psi_{i}^{r s}}{\psi_{i}^{2} 3^{r s}}, \frac{3\left(r^{3}-3 r^{2}+2 r\right)}{2} n\right),
\end{array}\right.
$$




$$
=\left\{\begin{array}{l}
\psi_{i}^{-2} \rho(l), \\
\psi_{i}^{s-2} \rho(l), \\
\psi_{i}^{r s-2} \rho(l), \\
\psi_{i}^{r s-2} \rho(l),
\end{array}\right.
$$

for all $l \in L$. The following cases hold with the below conditions:

$\mathbb{L}=3^{-2}$ if $i=0$ and $\mathbb{L}=3^{2}$ if $i=1$.

$\mathbb{L}=3^{s-2}$ for $s>2$ if $i=0$ and $\mathbb{L}=3^{2-s}$ if for $s 21$ if $i=1$.

$\mathbb{L}=3^{r s-2}$ for $s>2 / r$ if $i=0$ and $\mathbb{L}=3^{2-r s}$ if for $s<2 / r$ if $i=1$.

$\mathbb{L}=3^{r s-2}$ for $s>2 / r$ if $i=0$ and $\mathbb{L}=3^{2-r s}$ if for $s<2 / r$ if $i=1$.

Case $1 . \mathbb{L}=3^{-2}$ if $i=0$

$$
\begin{aligned}
\phi(\phi(t)-Q(l), n) & \leq \Phi^{\prime}\left(\frac{\mathbb{L}^{1-i}}{1-\mathbb{L}} \rho(l), n\right) \\
& =\Phi^{\prime}\left(\frac{3^{-2}}{1-3^{-2}} \frac{2 \varepsilon}{3\left(r^{3}-3 r^{2}+2 r\right)}, n\right) \\
& =\Phi^{\prime}\left(\varepsilon, \frac{24\left(r^{3}-3 r^{2}+2 r\right)}{2} n\right) .
\end{aligned}
$$

Case 2. $\mathbb{L}=3^{2}$ if $i=1$

$$
\begin{aligned}
\phi(\phi(t)-Q(l), n) & \leq \Phi^{\prime}\left(\frac{\mathbb{L}^{1-i}}{1-\mathbb{L}} \rho(l), n\right) \\
& =\Phi^{\prime}\left(\frac{1}{1-3^{3}} \frac{2 \varepsilon}{3\left(r^{3}-3 r^{2}+2 r\right)}, n\right) \\
& =\Phi^{\prime}\left(\varepsilon, \frac{-24\left(r^{3}-3 r^{2}+2 r\right)}{2} n\right) .
\end{aligned}
$$

Case 3. $\mathbb{L}=3^{s-2}$ for $s>2$ if $i=0$

$$
\begin{aligned}
\phi(\phi(t)-Q(l), n) & \leq \Phi^{\prime}\left(\frac{\mathbb{L}^{1-i}}{1-\mathbb{L}} \rho(l), n\right) \\
& =\Phi^{\prime}\left(\frac{3^{s-2}}{1-3^{s-2}} \frac{2 r \varepsilon\|l\|^{s}}{3\left(r^{3}-3 r^{2}+2 r\right) 3^{s}}, n\right) \\
& =\Phi^{\prime}\left(r \varepsilon\|l\|^{s}, \frac{3\left(r^{3}-3 r^{2}+2 r\right)}{2}\left(3^{2}-3^{s}\right) n\right) .
\end{aligned}
$$

Case $4 . \mathbb{L}=3^{2-s}$ for $s<2$ if $i=1$

$$
\begin{aligned}
\phi(\phi(t)-Q(l), n) & \leq \Phi^{\prime}\left(\frac{\mathbb{L}^{1-i}}{1-\mathbb{L}} \rho(l), n\right) \\
& =\Phi^{\prime}\left(\frac{1}{1-3^{2-s}} \frac{2 r \varepsilon\|l\|^{s}}{3\left(r^{3}-3 r^{2}+2 r\right) 3^{s}}, n\right) \\
& =\Phi^{\prime}\left(r \varepsilon\|l\|^{s}, \frac{3\left(r^{3}-3 r^{2}+2 r\right)}{2}\left(3^{2}-3^{s}\right) n\right) .
\end{aligned}
$$

Case 5. $\mathbb{L}=3^{r s-2}$ for $s>2 / r$ if $i=0$

$$
\begin{aligned}
\phi(\phi(t)-Q(l), n) & \leq \Phi^{\prime}\left(\frac{\mathbb{L}^{1-i}}{1-\mathbb{L}} \rho(l), n\right) \\
& =\Phi^{\prime}\left(\frac{3^{r s-2}}{1-3^{r s-2}} \frac{2 \varepsilon\|l\|^{r s}}{3\left(r^{3}-3 r^{2}+2 r\right) 3^{r s}}, n\right) \\
& =P h i^{\prime}\left(\varepsilon\|l\|^{r s}, \frac{3\left(r^{3}-3 r^{2}+2 r\right)}{2}\left(3^{2}-3^{r s}\right) n\right) .
\end{aligned}
$$

Case 6. $\mathbb{L}=3^{2-r s}$ for $s<2 / r$ if $i=1$

$$
\begin{aligned}
\phi(\phi(t)-Q(l), n) & \leq \Phi^{\prime}\left(\frac{\mathbb{L}^{1-i}}{1-\mathbb{L}} \rho(l), n\right) \\
& =\Phi^{\prime}\left(\frac{1}{1-3^{2-r s}} \frac{2 \varepsilon\|l\|^{r s}}{3\left(r^{3}-3 r^{2}+2 r\right) 3^{r s}} n\right) \\
& =\Phi^{\prime}\left(\varepsilon\|l\|^{r s}, \frac{3\left(r^{3}-3 r^{2}+2 r\right) 3^{r s}}{2}\left(3^{r s}-3^{2}\right) n\right) .
\end{aligned}
$$

Hence, the proof is completed.

\section{Data Availability}

No data were used to support this study.

\section{Conflicts of Interest}

The authors declare that there is no conflict of interest regarding the publication of this paper.

\section{Acknowledgments}

The second and fifth authors would like to express their sincere thanks to the financial and research support of Phuket Rajabhat University, 83000, Phuket, Thailand.

\section{References}

[1] S. M. Ulam, Problems in Modern Mathematics, Science Editions, Wiley, New York, 1964.

[2] D. H. Hyers, "On the stability of the linear functional equation," Proceedings of the National Academy of Sciences of the United States of America, vol. 27, pp. 222-224, 1941.

[3] S. M. Jung, "On the Hyers-Ulam-Rassias stability of a quadratic functional equation," Journal of Mathematical Analysis and Applications, vol. 232, pp. 384-393.1, 1999.

[4] H. A. Kenary, H. Rezaei, Y. Gheisari, and C. Park, "On the stability of set-valued functional equations with the fixed point alternative," Fixed Point Theory and Applications, vol. 2012, 17 pages, 2012.

[5] A. K. Mirmostafaee and M. S. Moslehian, "Fuzzy versions of Hyers-Ulam-Rassias theorem," Fuzzy sets and systems, vol. 159, pp. 720-729.1, 2008.

[6] M. S. Moslehian and T. M. Rassias, "Stability of functional equations in non-Archimedean spaces," Applicable Analysis and Discrete Mathematics, vol. 1, pp. 325-334, 2007. 
[7] K. Nikodem and D. Popa, "On single-valuedness of set-valued maps satisfying linear inclusions," Banach Journal of Mathematical Analysis, vol. 3, pp. 44-51, 2009.

[8] T. M. Rassias, "On the stability of the linear mapping in Banach spaces," Proceedings of the American Mathematical Society, vol. 72, pp. 297-300.1, 1978.

[9] V. Radu, "The fixed point alternative and the stability of functional equations," Fixed Point Theory, vol. 4, pp. 91-96, 2003, 1 .

[10] D. H. Hyers, G. Isac, and T. M. Rassias, Stability of Functional Equations in Several Variables, Birkhäuser, Boston, 1998.

[11] A. Bodaghi, "Intuitionistic fuzzy stability of the generalized forms of cubic and quartic functional equations," Journal of Intelligent \& Fuzzy Systems, vol. 30, pp. 2309-2317, 2016.

[12] S. M. Jung, Hyers-Ulam-Rassias Stability of Functional Equations in Nonlinear Analysis, Springer, New York, 2011.

[13] K. Cieplinski, "On the generalized Hyers-Ulam stability of multi-quadratic mappings," Computers \& Mathematcs with Applications, vol. 62, pp. 3418-3426.1, 2011.

[14] S. M. Jung, "On the Hyers-Ulam stability of the functional equations that have the quadratic property," Journal of Mathematical Analysis and Applications, vol. 222, pp. 126-137.1, 1998.

[15] Y. W. Lee, "On the stability of a quadratic Jensen type functional equation," Journal of Mathematical Analysis and Applications, vol. 270, pp. 590-601.1, 2002.

[16] C. G. Park, "On the Hyers-Ulam-Rassias stability of generalized quadratic mappings in Banach modules," Journal of Mathematical Analysis and Applications, vol. 291, pp. 214223.1, 2004.

[17] C. Park, H. A. Kenary, and T. M. Rassias, "Hyers-Ulam-Rassias stability of the additive-quadratic mappings in nonArchimedean Banach spaces," Journal of Inequalities and Applications, vol. 2012, 18 pages, 2012.

[18] H. Y. Shen and Y. Y. Lan, "On the general solution of a quadratic functional equation and its Ulam stability in various abstract spaces," Journal of Nonlinear Sciences and Applications, vol. 7, pp. 368-378, 2014.

[19] C. Dineshkumar, R. Udhayakumar, V. Vijayakumar, and K. S. Nisar, "A discussion on the approximate controllability of Hilfer fractional neutral stochastic integro-differential systems," Chaos, Solitons \& Fractals, no. article 110472, 2020.

[20] V. Vijayakumar and R. Udhayakumar, "Results on approximate controllability for non-densely defined Hilfer fractional differential system with infinite delay," Chaos, Solitons \& Fractals, vol. 139, article 110019, 2020.

[21] K. Kavitha, V. Vijayakumar, and R. Udhayakumar, "Results on controllability of Hilfer fractional neutral differential equations with infinite delay via measures of noncompactness," Chaos, Solitons \& Fractals, vol. 139, article 110035, 2020.

[22] M. Mohan Raja, V. Vijayakumar, R. Udhayakumar, and Y. Zhou, "A new approach on the approximate controllability of fractional differential evolution equations of order $1<\mathrm{r}<2$ in Hilbert spaces," Numerical Methods for Partial Differential Equations, Chaos, Solitons \& Fractals, vol. 141, article 110310, 2020.

[23] V. Vijayakumar and R. Udhayakumar, "A new exploration on existence of Sobolev-type Hilfer fractional neutral integrodifferential equations with infinite delay," Numerical Methods for Partial Differential Equations, vol. 37, pp. 750-766, 2020.
[24] N. Gunasekaran, G. Zhai, and Q. Yu, "Sampled-data synchronization of delayed multi-agent networks and its application to coupled circuit," Neurocomputing, vol. 413, pp. 499-511, 2020.

[25] N. Gunasekaran and G. Zhai, "Stability analysis for uncertain switched delayed complex-valued neural networks," Neurocomputing, vol. 367, pp. 198-206, 2020.

[26] N. Gunasekaran, N. M. Thoiyab, P. Muruganantham, G. Rajchakit, and B. Unyong, "Novel results on global robust stability analysis for dynamical delayed neural networks under parameter uncertainties," IEEE Access, vol. 8, pp. 178108178116, 2020.

[27] A. Mohanapriya, A. Ganesh, and N. Gunasekaran, "The Fourier transform approach to Hyers-Ulam stability of differential equation of second order," Journal of Physics: Conference Series, vol. 1597, article 012027, 2020.

[28] B. Unyong, A. Mohanapriya, A. Ganesh et al., "Fractional Fourier transform and stability of fractional differential equation on Lizorkin space," Advances in Difference Equations, vol. 2020, 23 pages, 2020.

[29] B. Unyong, V. Govindan, S. Bowmiya et al., "Generalized linear differential equation using Hyers-Ulam stability approach," AIMS Mathematics, vol. 6, pp. 1607-1623, 2021. 